
\title{
28 Research Square \\ Effect of Tellurium Doping on Optoelectronic Properties of Blue ZnTeSe Quantum Dots
}

\section{Eunjoo Jang ( $\nabla$ ejjang12@samsung.com )}

Samsung Advanced Institute of Technology https://orcid.org/0000-0003-2573-0176

\section{Taehyung Kim}

Samsung Advanced Institute of Technology https://orcid.org/0000-0002-7367-4941

Heejae Chung

Samsung Electronics

Seon-Myeong Choi

Samsung Electronics

Yu-Ho Won

Samsung Electronics

Jae-Yong Lee

Samsung Electronics

\section{Article}

Keywords:

Posted Date: January 26th, 2022

DOI: https://doi.org/10.21203/rs.3.rs-1183117/v1

License: (c) (i) This work is licensed under a Creative Commons Attribution 4.0 International License.

Read Full License 


\section{Abstract}

Quantum dot light emitting diodes (QD-LEDs) have high potential to be used in next-generation displays. The main challenge in the commercialisation of QD-LEDs is the improvement of the efficiency and stability of blue QD-LEDs. The most promising emitter for blue QD-LEDs is ZnTeSe QD, however it is concerned that tellurium causes spectral broadening and efficiency drop. In this work, we demonstrate the effect of Te clusters on the electronic structures of blue ZnTeSe QDs through first principle calculation. The photo dynamics at the ensemble and single dot level show that the lifting of hole-state degeneracy in Te-doped structures causes slow relaxation and peak emission shift. Furthermore, the strong confinement of hole wave functions in Te clusters improves the efficiency and stability of QD-LEDs by suppressing QD luminescence quenching under electrical bias. The understanding of the correlation between the photophysical nature of Te-doped QDs and device performance can provide a basis for designing blueemitting QD structures that are suitable for practical QD-LEDs.

\section{Introduction}

The development of efficient and stable blue-emitting fluorophores is one of the challenges for nextgeneration quantum dot light emitting diode (QD-LED) displays. Although QD-LEDs have numerous advantages over conventional technologies ${ }^{1,2}$, the performance of blue QD-LED devices is worse than that of red and green QD-LED devices ${ }^{3-5}$. This is partly due to the difficulties in synthesising high quality blue quantum dot (QD) materials, which intrinsically have more surface defects compared to red or green QD materials owing to the small size of CdSe or $\ln \mathrm{P}^{4,6}$. ZnSe QDs have a wide energy band gap. Thus, it is necessary to add a small amount of tellurium $(\mathrm{Te})$ to tune the emission at a blue wavelength of 460 $\mathrm{nm}$. Numerous works have investigated ZnTeSe QDs, and highly efficient blue QDs with near-unity photoluminescence $(\mathrm{PL})$ quantum yield $(\mathrm{QY})$ have been recently reported ${ }^{7}$. However, still there are doubts on using Te for the blue QDs based on the expectations that it is more vulnerable to oxygen than selenium (Se) and induces multiple trap states that increase the spectral width and decrease the $\mathrm{QY}^{8}$. Thus, to design blue QD structures that are suitable for LED applications, it is necessary to understand the role of Te doping in ZnTeSe with respect to its photonic properties and correlation with device performance. There is no strong relationship between the PL QY and external quantum efficiency (EQE) of state-of-the-art QD-LEDs because the current EQE strongly depends on the electron/hole $(\mathrm{e} / \mathrm{h})$ charge balance rather than the luminescence of $Q D s^{9,10}$. In addition, previous studies have reported that it is critical to alleviate the accumulation of charges inside QDs by preparing a thick shell or alloy shell for achieving a high PL QY, particularly under multi-exciton generation conditions ${ }^{11,12}$. Therefore, to develop highly efficient and stable blue QD-LEDs, we believe that it is important to address the issues of e/h behaviours such as e/h overlap under bias, exciton recombination inside ZnSe-based QDs, and their complex interactions with adjacent conducting layers in LED devices. In this work, we examine the role of Te doping in blue-emitting ZnTeSe QDs to create highly efficient and stable electroluminescent (EL) devices and investigate the origin of the improvements in the device performances. The electronic structures of Te-doped ZnTeSe QDs significantly influence PL and EL properties by forming localised hole 
states close to the valence band maximum (VBM); this eventually enhances the quantum confinement effect. The spatial clustering effect of Te in ZnTeSe QDs is systematically shown via density functional theory (DFT) calculations. Additionally, hole relaxation properties of blue emitting ZnTeSe QDs are experimentally confirmed by measuring transient absorption (TA) dynamics and photo dynamics at the ensemble and single dot levels for the first time. It is newly suggested that Te-induced localised hole states facilitate single exciton recombination by suppressing PL quenching under a strong electric field. Furthermore, the EL spectral shift of the blue ZnTeSe based QD-LEDs is compared with the changes in the $\mathrm{PL}$ of the QDs with different Te concentrations at similar exciton densities.

\section{Zntese Qds With Localised Te States}

ZnTeSe cores with Te concentrations of $0 \%, 3 \%, 5 \%$, and 7\% and respective ZnTeSe/ZnSe/ZnS core/multi-shell QDs were prepared according to previously introduced methods ${ }^{13,14}$. Controlled amounts of Te and a Se precursor were simultaneously added during the preparation of a core. Then, the $\mathrm{ZnSe} / \mathrm{ZnS}$ shell was grown on the core in the presence of halide additives. Figure $1 \mathrm{a}$ shows the schematic and high-resolution transmission electron microscopy (HR-TEM) image of a ZnTeSe/ZnSe/ZnS QD and the diffraction pattern observed in the [100] direction. All the QDs showed pure zinc blende crystalline structures (Supplementary Figure 1), and the core/shell QDs exhibited high PL QYs of $93-97 \%$ with average sizes of $\sim 10 \mathrm{~nm}$ (Supplementary Figure 2). The detailed optical properties, elemental compositions, and structural information of the QDs are presented in Supplementary Table 1. As previously reported ${ }^{15,16}$, the most noticeable effect of Te doping on the optical properties of the ZnTeSe/ZnSe/ZnS QDs was the red shift in the PL emission from $440 \mathrm{~nm}$ to $461 \mathrm{~nm}$ and an increase in the spectral width from $12 \mathrm{~nm}$ to $36 \mathrm{~nm}$ as the Te concentration increased to 7\% (Supplementary Figure 3). This was because of the narrowing of the energy band gap due to the contribution of the ZnTe energy level. However, the concurrent changes in the Stokes shifts (from $2 \mathrm{~nm}$ to $8 \mathrm{~nm}$ ) and average transient PL lifetimes (from $18 \mathrm{~ns}$ to $55 \mathrm{~ns}$ ) with the Te concentration (Supplementary Table 1) implied that the energy transitions in the electronic structures of the ZnTeSe QDs were fundamentally altered from the simple combination of the ZnSe and ZnTe electronic structures. In CdSe doped with a few Te substitutional impurities, the Stokes shift, radiative decay time, and biexciton binding energy depended on the QD size owing to the carrier localisation around the Te dopant ${ }^{2}$. We used the density functional method and the projector-augmented wave pseudopotential provided by the Vienna ab initio simulation package to understand the electronic structure and carrier localisation effect of Te doping ${ }^{17}$. The size of atomic clusters and the number of Te dopants in ZnTeSe QDs were similar to experimental values. Specifically, for the ZnSe core, an atomic cluster model with a diameter of $3.6 \mathrm{~nm}$ was constructed with $532 \mathrm{Zn}$ atoms and 555 Se atoms, and we randomly placed Te atoms at Se positions in the Te-doped structures. Figure $1 \mathrm{~b}$ shows the representative atomic models of the ZnSe and ZnTeSe core QDs with 7\% Te and the corresponding energy levels close to the band edges. Te doping induced an upshift of the VBM while maintaining conduction band minimum (CBM). Furthermore, the clustering size (number of Te atoms in a cluster) affected the degree of changes in the VBM. We calculated the energy band gap of ZnTeSe QDs at different Te concentrations and clustering sizes and compared it with experimental values (Figure 1c). 
For each given Te concentration, band gap calculations were performed for selected Te distributions with high probability among randomly generated 30,000 atomic configurations. The maximum number of $\mathrm{Te}$ atoms per cluster determined energy band gap, which accorded to the experimental values changed from $3.36 \mathrm{eV}$ to $3.21 \mathrm{eV}$. The locations and shapes of Te clusters also affected band gap to result in the discrepancies of calculated values for same sized cluster (Supplementary Data 1). The calculated oscillator strength and absorbance spectra also showed the same trend of band gap decrease, and the degeneracy close to the lowest states was lifted (Supplementary Figure 4). Moreover, the discrete energy states close to the VBM developed more as the clustering size increased with the Te concentration. Therefore, the localised Te energy level of ZnTeSe QDs can play an important role in determining photophysical and EL properties.

The effect of the Te cluster on the energy transition was verified by measuring the fast temporal evolutions of TA dynamics as a function of the Te concentration in ZnSeTe/ZnSe/ZnS core/shell QDs (Figure 1d). To ensure the measurement of relaxation without accumulated charges, pump fluence was controlled at a low excitation level $(50 \mu \mathrm{W})$, which corresponded to an average exciton occupancy $\left\langle\mathrm{N}_{\mathrm{x}}\right\rangle$ of 0.5. Consecutive measurements were performed with $(A)$ and without $\left(A_{0}\right)$ a pump pulse to obtain the transient absorbance difference, $\triangle \mathrm{A}=\mathrm{A}-\mathrm{A}_{0}$, which represented the population of excited carriers at a given energy in the delay time between the pump and probe pulses. The photo generated hot carriers in QDs relaxed to the band-edge state within a few picoseconds, which was accompanied by a rise and decay in TA spectra. Figure 1e shows the temporal evolutions of $\triangle \mathrm{A}$ for the $\mathrm{ZnSeTe} / \mathrm{ZnSe} / \mathrm{ZnS}$ core/shell QDs with $0 \%, 3 \%, 5 \%$, and 7\% Te. In ZnSe QDs, a distinct band structure was observed in a short period ( $t$ $\sim 0.5 \mathrm{ps}$ ), and the positions of each transition matched with the second derivative of the absorption spectra (Supplementary Figure 3). The three lowest transitions in ZnSe were assigned as $1 S_{e^{-}}-1 S_{h}, 1 S_{e^{-}}$ $2 S_{h}$, and $1 \mathrm{P}_{\mathrm{e}}-1 \mathrm{P}_{\mathrm{h}}{ }^{18-20}$. After photoexcitation at $3.1 \mathrm{eV}$, hot carriers initially populated the $1 \mathrm{P}_{\mathrm{e}}-1 \mathrm{P}_{\mathrm{h}}$ state and decayed with the build-up of the $1 S_{e}-2 S_{h}$ bleach and $1 S_{e}-1 S_{h}$ bleach signals for $0 \%$ Te $(426 \mathrm{~nm}$ and $442 \mathrm{~nm}$, respectively). During hot-carrier relaxation below $5 \mathrm{ps}$, the $1 \mathrm{~S}_{\mathrm{e}}-1 \mathrm{~S}_{\mathrm{h}}$ transition at the longer wavelength progressively increased more than the $1 \mathrm{~S}_{\mathrm{e}}-2 \mathrm{~S}_{\mathrm{h}}$ transition owing to the hole-relaxation process. Interestingly, the position of the $1 S_{\mathrm{e}}-1 \mathrm{~S}_{\mathrm{h}}$ transition gradually faded into a shoulder-like feature and converted into a broadened red-shift spectrum as the Te-doped state continued to grow. Moreover, the build-up time of the $1 \mathrm{~S}_{\mathrm{e}}-1 \mathrm{~S}_{\mathrm{h}}$ bleach signal increased as with the Te concentration (Supplementary Figure 5), indicating that more localised hole states formed above the VBM of ZnSe as the Te concentration increased.

We further investigated the optical properties of individual QDs to exclude the effect of distributions. Figure $2 \mathrm{a}$ shows the PL spectra of a representative single dot (average and $1 \mathrm{~s}$ frame) and the ensemble for the ZnTeSe/ZnSe/ZnS QDs with different Te concentrations. For all samples, the shapes of the accumulated spectra of more than 30 single dots were almost identical to that of the respective ensemble (Supplementary Figure 6). It is worth noting that the spectral width of the ensemble and single dot PL spectra increased with the Te concentration (Figure 2b). This implied that the major reason for the spectral broadening was Te-induced hole states, regardless of heterogeneous particle distribution or 
spectral diffusion. Furthermore, the PL lifetime of the ensemble and single dot decreased with the peak energy owing to the localised Te states (Figure 2c). As these characteristics of single dot analysis were the same as those observed in ensembles, it was clear that localised Te states were the origin of the spectral modulations instead of the heterogeneity of particles. In addition, the surface defect emission for Te-doped ZnTeSe core QDs (Supplementary Figure 3) was considerably suppressed at low temperatures, which suggested strong spatial confinement in the core owing to localised Te states.

\section{Effect Of Localised Te States On Qd-leds}

The effect of the Te-induced energy levels on the QD-LEDs was investigated by fabricating unit devices using the ZnTeSe/ZnSe/ZnS QDs with $0 \%, 3 \%, 5 \%$, and 7\% Te (Figure 3a). The EQE of the QD-LEDs was $8.9 \%, 9.8 \%, 11.6 \%$, and $14.4 \%$ at Te concentrations of $0 \%, 3 \%, 5 \%$, and $7 \%$, respectively, even though all the QDs had similar PL QYs (Figure 3b). Furthermore, the current densities of the devices increased with the Te concentration (Supplementary Figure 8), indicating that heavily Te-doped QDs could accommodate more charges and recombine them more efficiently. (The brightness for the devices with $7 \%$ and $0 \% \mathrm{Te}$ was $93,915 \mathrm{~cd} \mathrm{~m}^{-2}$ and 39,140 $\mathrm{cd} \mathrm{m}^{-2}$, respectively. Supplementary Figure 8) The operation lifetime increased with the EQE (Supplementary Figure 8). Another notable feature was that the EQE of the devices with heavily Te-doped QDs decreased more rapidly after it reached the maximum value. This could be because of the excessive charge injection due to the Te doping. Figure $3 \mathrm{~d}$ shows the impedance analysis based on the capacitance-voltage $(C-V)$ relationships. As the carriers were injected, the capacitance increased and reached a maximum, and then, it sharply decreased as excitons recombined. The increase and decrease in the capacitance occurred faster as Te doping increased, indicating that charge injection and recombination were accelerated by the Te-induced hole states. Furthermore, the peak capacitance (built-up charges) decreased as the Te concentration increased. The photoelectron yield spectra showed that the VBM shifted from $5.44 \mathrm{eV}$ to $5.35 \mathrm{eV}$ as the Te concentration increased from 0$7 \%$ (Supplementary Figure 8). This difference agreed with the change in the emission peak ( 128 meV). Hole-only devices (HODs) and electron-only devices (EODs) consisting of the ZnTeSe/ZnSe/ZnS QDs with various Te concentrations revealed that the hole and electron current densities at $8 \mathrm{~V}$ increased by 2 times as the Te concentration increased from 0-7\% (Supplementary Figure 9). This suggested that the hole injection facilitated by the Te-induced low lying VBM reinforced the electron injection by the induced electric field to lead to more efficient exciton recombination ${ }^{21}$. We further investigated the photophysical properties of the QDs under electrical bias. The PL spectra of QD emitting layers were measured at low voltages ( $<\sim 2.5 \mathrm{~V}$ ) before the QD-LEDs showed electroluminescence (Figure 3c). The PL intensity of ZnSe QDs decreased the most by 20\%, although the QD with 7\% Te maintained the initial value under bias. In addition, the degree of the peak shift (Supplementary Figure 10) increased with the Te concentration. This could be because weakly bound holes at the valence edge of ZnSe transferred to adjacent conducting layers under the electric field, causing easier non-radiative recombination compared to strongly bound localised Te hole states. The effective mass approximation calculations performed considering the external electric field also verified that the e/h overlap of the QD with 7\% Te was maintained better compared to ZnSe QDs under the electric field (Figure 3e and 3f). 
The dependency of PL on the Te concentration could be interpreted as a quantum-confined Stark effect (QCSE), which typically causes the electric field dependence of the peak shift and emission intensity change ${ }^{22,23}$. The contribution of the QCSE was examined by creating a capacitor structure (Figure $4 \mathrm{a}$ ) to strictly allow the electric field effect without charge flow. As the Te-doped states had a negligible effect on the spectral responses, we could confirm that the QCSE was not responsible for the spectral transitions. Two other kinds of semicapacitor devices were fabricated to distinguish between the effects of holes (Figure $4 \mathrm{~b}$ ) and electrons (Figure 4c). Interestingly, the trend of the PL changes in the capacitive EOD (ITO/PMMA/QD/ZnMgO/Al) was the same as that in the LEDs, and the capacitive HOD (ITO/PEDOT/TFB/QD/PMMA/Al) did not show this trend. This indicated that the charge interaction between the QD and electron transport layer (ETL) dominated the PL changes and EQE of the QD-LEDs and that localised Te states restricted hole transfer to the ETL, which suppressed nonradiative recombination. As electrons tend to pile up at the QD/ETL interface owing to the large band gap of blue $\mathrm{QDs}^{5}$, a strong electric field could push holes toward the piled-up electrons, which makes nonradiative recombination more likely. Therefore, localised hole states are advantageous for suppressing biasinduced PL quenching.

The localised Te-hole states affected the EL spectral features of the QD-LEDs under multi-exciton generation conditions. Although easily generated multi-excitons in QD-LEDs can cause the efficiency rolloff ${ }^{11}$ or degradation of devices ${ }^{24}$, the definition of the multi-exciton regime during QD-LED operation is not clear. We applied power dependent spectroscopic analyses to evaluate the exciton densities in the QD-LEDs according to the peak wavelength shift. Figures $5 \mathrm{a}$ and $5 \mathrm{~b}$ show the trend of the PL peak shifts of the ZnTeSe/ZnSe/ZnS QDs with 7\% Te as a solution and film with representative spectra under a range of optical excitation fluxes. The PL peaks of the QD solution were $468 \mathrm{~nm}(2.65 \mathrm{eV})$ and $464 \mathrm{~nm}$ $\left(2.67 \mathrm{eV}\right.$ ) at exciton occupancies $<N>$ of 0.01 and 0.52 , respectively ${ }^{25,26}$ (Supplementary Table 4), and the QD film blue-shifted from $466 \mathrm{~nm}(2.66 \mathrm{eV})$ to $459 \mathrm{~nm}(2.70 \mathrm{eV})$ as the excitation power changed from $0.01 \mu \mathrm{W}$ to $36 \mu \mathrm{W}$. The EL spectra also blue-shifted from $470 \mathrm{~nm}(2.64 \mathrm{eV})$ to $461 \mathrm{~nm}(2.69 \mathrm{eV})$, and the spectral width decreased as the applied bias increased from $2.8 \mathrm{~V}$ to $8 \mathrm{~V}$ (Figure 5c). The PL and EL spectral changes with the exciton density strongly depended on the Te concentration. Furthermore, the same effect of Te doping was observed on the wavelength shift of single dot PL (Supplementary Figure 13). As PL and EL spectral behaviours were quantitatively similar, the exciton density of the QD-LEDs could be derived from the correlation obtained in the power dependent PL measurements (Supplementary Figure 14). The QD-LEDs could hold multi-excitons over $\sim 3.7 \mathrm{~V}$ with a current density of $150 \mathrm{~mA} / \mathrm{cm}^{2}$ (dashed line in Figure 5c). As the corresponding brightness of the blue QD-LEDs with 7\% Te was 17,000 $\mathrm{cdm}^{-2}$ at $3.7 \mathrm{~V}$, it was presumed that the practical operation of commercial products might not require multi-exciton generation conditions. A previous study reported the spectral blue-shift in the multi-exciton region was induced by strong Coulomb repulsion between confined holes in the biexciton state ${ }^{27}$.

\section{Conclusion}


Highly luminescent ZnTeSe/ZnSe/ZnS core/multi-shell QDs were prepared with $0 \%, 3 \%, 5 \%$, and 7\% Te in the core. The effect of Te clusters on the electronic structures of the QDs was examined through DFT calculations. The photo dynamics at the ensemble and single dot levels revealed that Te clusters formed localised hole states. The Te clusters in ZnTeSe-based QDs improved the efficiency of the QD-LEDs by suppressing QD luminescence quenching under electrical bias because of strongly bound localised hole states. Furthermore, quantitative analysis showed that localised Te-hole states affected the EL spectral features of the QD-LEDs under multi-exciton generation conditions. Our results provide a fundamental understanding of ZnTeSe-based blue QDs for practical application to QD-LED displays.

\section{Methods}

Materials. Diethylzinc ( $\mathrm{Et}_{2} \mathrm{Zn}, 1.0 \mathrm{M}$ in hexanes), selenium (Se, 99.99\%), tellurium (Te, 99.999\%), diphenylphosphine (DPP, 98\%), zinc acetate $\left(\mathrm{Zn}(\mathrm{OAc})_{2}, 99.99 \%\right)$, zinc acetate dihydrate $\left(\mathrm{Zn}(\mathrm{OAc})_{2} \cdot 2 \mathrm{H}_{2} \mathrm{O}\right.$, $99 \%)$, magnesium (II) acetate tetrahydrate $\left(\mathrm{Mg}(\mathrm{OAC})_{2} \cdot 4 \mathrm{H}_{2} \mathrm{O}, 99 \%\right)$, tetramethylammonium hydroxide pentahydrate ( $\left.\mathrm{TMAH} \cdot 5 \mathrm{H}_{2} \mathrm{O}, 97 \%\right)$, sulphur ( $\left.\mathrm{S}, \geq 99.99 \%\right)$, zinc chloride $\left(\mathrm{ZnCl}_{2}\right.$, anhydrous, $\left.99.95 \%\right)$, hydrofluoric acid (HF, $48 \mathrm{wt} \%$ in water), oleic acid (OA, 90\%), oleylamine (OAm, technical grade, 70\%), tri-noctylamine (TOA, $\geq 99.0 \%$ ), hexane (anhydrous, 99.8\%), toluene (anhydrous, 99.8\%), 1,2-dimethylbenzene (o-xylene, anhydrous, 97\%), n-octane (anhydrous, $\geq 99 \%$ ), poly (methyl methacrylate) (PMMA, average Mw $=97,000)$, and polyvinylpyrrolidone (PVP, average Mw = 40,000) were purchased from Sigma-Aldrich. Trioctylphosphine (TOP, 97\%) was purchased from Strem Chemicals. Acetone (99.7\%), isopropanol (IPA, 100\%), and ethanol (HPLC, 99.9\%) were purchased from Samchun Chemicals. Dimethyl sulfoxide (DMSO, 99.5\%) and ethyl acetate (99\%) were obtained from Daejung Chemicals. Tris(4-carbazoyl-9ylphenyl)amine (TCTA) and 1,4,5,8,9,11-hexa-azatriphenylene hexacarbonitrile (HATCN) were purchased from Lumtech and LGChem, respectively.

Preparation of precursor. $0.4 \mathrm{M}$ zinc oleate $\left(\mathrm{Zn}(\mathrm{OA})_{2}\right)$ was prepared by adding $48 \mathrm{mmol}$ of $\mathrm{Zn}(\mathrm{OAc})_{2}$ and $96 \mathrm{mmol}$ of $\mathrm{OA}$ in $120 \mathrm{ml}$ of TOA and evacuating the mixture at $120^{\circ} \mathrm{C}$ for $30 \mathrm{~min}$. Then, the mixture was heated to $280^{\circ} \mathrm{C}$ for $10 \mathrm{~min}$ in a $\mathrm{N}_{2}$ atmosphere. $2 \mathrm{M} \mathrm{Se} / \mathrm{TOP}, 1 \mathrm{M} \mathrm{S} / \mathrm{TOP}$, and $0.1 \mathrm{M} \mathrm{Te} / \mathrm{TOP}$ were prepared by separately dissolving Se pellets $(7.90 \mathrm{~g}), \mathrm{S}$ powder $(1.60 \mathrm{~g})$, and Te pellets $(0.64 \mathrm{~g})$ in $50 \mathrm{ml}$ TOP and heating each solution to $120^{\circ} \mathrm{C}$ for $30 \mathrm{~min}$ in a $\mathrm{N}_{2}$-filled glove box.

Synthesis of ZnTeSe core. $13 \mathrm{mmol}$ of OA and $9 \mathrm{mmol}$ of OAm were mixed in $50 \mathrm{~mL}$ of TOA in a $250 \mathrm{ml}$ flask with stirring and evacuated at $120^{\circ} \mathrm{C}$ for $30 \mathrm{~min}$. Then, the mixture was heated to $240^{\circ} \mathrm{C}$ in a $\mathrm{N}_{2}$ atmosphere. $\mathrm{Et}_{2} \mathrm{Zn}(9 \mathrm{mmol})$ was rapidly injected into the reaction solution. Then, a mixture of $2 \mathrm{M}$ $\mathrm{Se} / \mathrm{TOP}$ (4.5 mmol), 0.1 M Te/TOP, and DPP ( $4.5 \mathrm{mmol})$ was rapidly added under $\mathrm{N}_{2}$ flow $(800 \mathrm{cc} / \mathrm{min}) .0$ $\mathrm{mmol}, 0.14 \mathrm{mmol}, 0.23 \mathrm{mmol}$, and $0.30 \mathrm{mmol}$ of $0.1 \mathrm{M} \mathrm{Te} / \mathrm{TOP}$ were added to synthesise ZnTeSe cores with $0 \%, 3 \%, 5 \%$, and $7 \%$ Te, respectively. The reaction medium was maintained with vigorous stirring at $300^{\circ} \mathrm{C}$ for $40 \mathrm{~min}$. The $\mathrm{ZnTeSe}$ cores were precipitated by the addition of ethanol followed by centrifugation at 6,000 rpm for $5 \mathrm{~min}$ and redispersion in hexane. 
Synthesis of ZnTeSe/ZnSe/ZnS QDs. In a $250 \mathrm{ml}$ flask, $4.8 \mathrm{mmol}$ of $\mathrm{Zn}(\mathrm{OAc})_{2}$ and $9.6 \mathrm{mmol}$ of OA were mixed with $80 \mathrm{~mL}$ of the TOA solvent with stirring, and the mixture was evacuated at $120^{\circ} \mathrm{C}$ for $15 \mathrm{~min}$. Then, the mixture was heated to $280^{\circ} \mathrm{C}$ to prepare the $\mathrm{Zn}(\mathrm{OA})_{2}$ precursor under $\mathrm{N}_{2}$ flow $(800 \mathrm{cc} / \mathrm{min})$ and cooled to $220^{\circ} \mathrm{C}$. The ZnTeSe core (optical density (OD) at 100 -fold dilution was 0.54 at the first absorption peak, $8 \mathrm{ml}$ ) was rapidly injected into the $\mathrm{Zn}(\mathrm{OA})_{2}$ precursor in TOA. Then, $\mathrm{HF}(10 \mathrm{vol} \% \mathrm{in}$ acetone, $0.44 \mathrm{ml}$ ) and $\mathrm{ZnCl}_{2}$ (10 $\mathrm{wt} \%$ in acetone, $0.09 \mathrm{mmol}$ ) were added to the reaction mixture, and the temperature was increased to $340^{\circ} \mathrm{C}$. The $\mathrm{ZnSe}$ mid-shell was grown by adding $18 \mathrm{mmol}$ of $0.4 \mathrm{M}$ $\mathrm{Zn}(\mathrm{OA})_{2}$ and $12 \mathrm{mmol}$ of $0.4 \mathrm{M} \mathrm{Se} / \mathrm{TOP}$ at $340^{\circ} \mathrm{C}$ to the reaction mixture and reacting for $1 \mathrm{~h}$. The $\mathrm{ZnS}$ shell was grown by adding $9.6 \mathrm{mmol}$ of $0.4 \mathrm{M} \mathrm{Zn}(\mathrm{OA})_{2}$ and $22.4 \mathrm{mmol}$ of $1 \mathrm{M} \mathrm{S} / \mathrm{TOP}$ to the reaction mixture and further reacting for $1.5 \mathrm{~h}$.

Synthesis of $\mathrm{ZnMgO}$ nanoparticles. $\mathrm{Zn}_{0.85} \mathrm{Mg}_{0.15} \mathrm{O}$ nanoparticles were synthesised by dissolving 8.5 mmol of $\mathrm{Zn}(\mathrm{OAc})_{2} \cdot \mathrm{H}_{2} \mathrm{O}$ and $1.5 \mathrm{mmol}$ of $\mathrm{Mg}(\mathrm{OAc})_{2} \cdot 4 \mathrm{H}_{2} \mathrm{O}$ in $100 \mathrm{~mL}$ of DMSO. In a separate vial, 14.7 mmol of TMAH $5 \mathrm{H}_{2} \mathrm{O}$ was dissolved in $30 \mathrm{~mL}$ of ethanol, and then, the solution was added to the DMSO solution including $\mathrm{Zn}$ and $\mathrm{Mg}$ precursors and stirred for $1 \mathrm{~h}$ at room temperature $\left(25^{\circ} \mathrm{C}\right)$. The $\mathrm{ZnMgO}$ nanoparticles were collected by adding ethyl acetate. They were washed with ethanol and ethyl acetate, followed by centrifugation at $6000 \mathrm{rpm}$. Finally, the precipitate was dispersed in ethanol.

Material characterisation. The absorption and PL spectra of the QDs were measured by employing a UVvis spectrometer (Cary 5000, Agilent) and a fluorescence spectrophotometer (F7000, Hitachi), respectively. The low temperature PL spectra were measured at a liquid nitrogen temperature using an equipped low temperature accessory. The PL QY was determined using an absolute PL QY spectrometer (Quantaurus-QY, Hamamatsu). The power-dependent PL spectra of the QDs were measured using a confocal microscope with a circularly polarised picosecond-pulsed excitation laser of $395 \mathrm{~nm}$ (LDH-D-C390, Picoquant) with a repetition rate of $1 \mathrm{MHz}$. The laser beam was focused on the dry-type objective (UplanSApo, $0.16 \mathrm{NA}, 4 \mathrm{x}$, Olympus). The collected photons were reflected by a dielectric mirror (MRA12E02, Thorlabs), and they entered the input slit of a dual-port monochromator (Kymera 193i-B, Andor), which was directly connected to a charge coupled device (iVac 316, Andor) and photon counting detectors (PMA hybrid 40, Picoquant). Transmission electron microscopy (TEM) analysis was performed using a Titan ChemiSTEM electron microscope operated at $200 \mathrm{keV}$. The inductively coupled plasma (ICP) atomic emission spectroscopy analysis of Zn, Se, Te, and S was performed using ICPS-8100 (Shimadzu). X-ray diffraction (XRD) patterns were recorded using a diffractometer (D8 Advance, Bruker) with a Cu-Ka source. The ionisation potential was measured by a photoelectron spectrophotometer (AC3, Riken Keiki) in air. Time-resolved absorption spectra were obtained using an ultrafast pump-probe spectroscopy system containing a Ti:sapphire regenerative amplifier (Libra, Coherent), optical parametric amplifier (TOPAS, Light conversion), and transient absorption (TA) spectrometer (Helios, Ultrafast Systems). A part of the output of the amplifier (pulse duration $=50 \mathrm{fs}$; a repetition rate $=1 \mathrm{KHz}$; centre wavelength $=800 \mathrm{~nm}$ ) was used to pump the optical parametric amplifier that delivered optical pump pulses at $400 \mathrm{~nm}$ for TA measurements. The pump pulses were chopped at $500 \mathrm{~Hz}$, and the probe spectra were directed to a fast CMOS array detector. All TA spectra were measured at a pump power of ca. 100 
$\mu \mathrm{W}$ to suppress any undesired multi-exciton effects. All measurements were carried out in a vigorously stirred octane-dispersed QD solution.

Single-dot measurement. Octane-dispersed QDs were highly diluted with a $1 \mathrm{wt} \%$ PMMA toluene solution and spin coated onto a cleaned glass coverslip at a speed of $3000 \mathrm{rpm}$ for $60 \mathrm{~s}$. The QD-coated coverslip was encapsulated with an epoxy resin (s-209, Devcon). Single-dot measurement was performed using a laser scanning confocal microscope with a circularly polarised picosecond-pulsed excitation laser of 395 $\mathrm{nm}$ (LDH-D-C-390, Picoquant) with a repetition rate of $1 \mathrm{MHz}$. In the setup, a scanning galvo-mirror was used to obtain an image from different sample areas. The laser beam was focused on the oil immersion objective (UplanSApo, 1.4 NA, 100x, Olympus). The collected photons were split by a nonpolarised cube beam splitter. They entered the input slit of a polychromator (Kymera 193i, Andor), which was directly connected to an electron multiplying charge coupled device (ixon897, Andor) and the active area of a single photon avalanche photodiode (PDM series, MPD). Detected single-dot photons were registered by a time-correlated single photon counting card (TCSPC, TimeHarp 260, Picoquant), which operated in the first-in-first-out regime. The full width at half maximum (FWHM) of the overall instrumental response function was approximately $400-500$ ps. The Symphotime 64 software (Picoquant) was used for data acquisition and processing.

Device fabrication and characterisation. Patterned indium tin oxide (ITO) was coated on glass substrates (Techno Print Co., Ltd., sheet resistance $\sim 10 \mathrm{ohm} /$ square, 2 inch $\times 2$ inch, ITO thickness $=150 \mathrm{~nm}$ ), which were washed with IPA in an ultrasonic bath. Then, the substrates were dried and exposed to UV-ozone treatment for $20 \mathrm{~min}$ (Jelight, UV0144AX-220). A poly(ethylenedioxythiophene):polystyrenesulfonate (PEDOT:PSS, Heraeus Clevios, Al 4083) solution was spin coated on the ITO glass at $2200 \mathrm{rpm}$ for $50 \mathrm{~s}$ and annealed at $150^{\circ} \mathrm{C}$ for $10 \mathrm{~min}$. Subsequently, the samples were transferred to a $\mathrm{N}_{2}$-filled glove box and annealed at $150^{\circ} \mathrm{C}$ for $30 \mathrm{~min}$. Poly(9,9-dioctyl-fluorene-co-N-(4-(3-methylpropyl)) diphenylamine (TFB, Sumitomo Chemical, Mw 300,000) dissolved in o-xylene (0.7 wt\%) was spin coated at $2000 \mathrm{rpm}$ for $40 \mathrm{~s}$ and baked at $150^{\circ} \mathrm{C}$ for $30 \mathrm{~min}$. QDs were dispersed in octane ( 3 wt\%), spin coated at $3000 \mathrm{rpm}$ for $40 \mathrm{~s}$, and baked at $80^{\circ} \mathrm{C}$ for $30 \mathrm{~min}$. A solution of $\mathrm{ZnMgO}$ nanoparticles $(12 \mathrm{~mol} \% \mathrm{Mg}, 70 \mathrm{mg} / \mathrm{mL}$ in ethanol) was spin coated at $4000 \mathrm{rpm}$ for $10 \mathrm{~s}$ and annealed at $80^{\circ} \mathrm{C}$ for $30 \mathrm{~min}$. The thicknesses of the QDs and ZnMgO layers was controlled by the spin speed to match the target values calibrated via ellipsometry and TEM analysis. The substrates were transferred to a vacuum chamber $\left(<5 \times 10^{-7}\right.$ Torr $)$ to deposit an aluminium cathode layer through thermal evaporation at a rate of $1 \AA \mathrm{s}^{-1}$. Finally, the device was encapsulated with a cover glass. A hole-only device was prepared with the structure of ITO (150 $\mathrm{nm}) /$ PED OT:PSS $(30 \mathrm{~nm}) /$ TFB $(25 \mathrm{~nm}) / \mathrm{QD} / \mathrm{TCTA}(36 \mathrm{~nm}) / \mathrm{HATCN}(10 \mathrm{~nm}) / \mathrm{Ag}(100 \mathrm{~nm})$. All layers were processed using the abovementioned method except TCTA and HATCN, which were deposited through thermal evaporation. An electron-only device was prepared with the structure of ITO $(150 \mathrm{~nm}) / \mathrm{ZnMgO}(20$ $\mathrm{nm}) / \mathrm{QD} / \mathrm{ZnMgO}(20 \mathrm{~nm}) / \mathrm{Al}(100 \mathrm{~nm})$ via spin coating. The capacitor devices were fabricated using PMMA and PVP as insulating layers with a structure of ITO (150 nm)/PMMA (50 nm)/QD/PVP (50 $\mathrm{nm}) / \mathrm{Al}(100 \mathrm{~nm})$. A $0.5 \mathrm{wt} \%$ PMMA ethanol solution and $1.0 \mathrm{wt} \%$ PVP acetone solution were used to create $50 \mathrm{~nm}$ thick layers through spin coating. Two types of semi-insulating devices were fabricated with 
structures of ITO (150 nm)/PEDOT:PSS (30 nm)/TFB (20 nm)/QD/PMMA (50 nm)/Ag (100 nm) and ITO $(150 \mathrm{~nm}) / \mathrm{PMMA}(50 \mathrm{~nm}) / \mathrm{QD} / \mathrm{ZnMgO}(20 \mathrm{~nm}) / \mathrm{Al}(100 \mathrm{~nm})$. Device performance was determined using a CS-2000A spectroradiometer (Minolta) and source meter (Keithley 2635B). The impedance spectra of the devices were measured using the SP-300 impedance analyser (Biologic). The voltage dependent PL spectra of the devices were measured by illuminating a pixel with a $405 \mathrm{~nm}$ continuous wave laser (Power Technology Inc.).

Ab-initio calculation. The $\mathrm{ZnSe}$ core with a diameter of $3.6 \mathrm{~nm}$ consisted of $523 \mathrm{Zn}$ and $555 \mathrm{Se}$ atoms. The number of Te atoms in the ZnTeSe cores with $3 \%, 5 \%$, and $7 \%$ Te was 17, 28, and 39, respectively. The energy levels and oscillator strength for optical transitions in the ZnTeSe cores were calculated using the pseudopotential density functional method with a plane-wave basis set. The exchange correlation of electrons was treated within the Perdew-Burke-Ernzerhof generalised gradient approximation revised for solids. The cut-off energy for the expansion of wave functions and potentials in the plane-wave basis was set as $300 \mathrm{eV}$. We used the projector augmented wave pseudopotentials provided by the Vienna $a b$ initio simulation package. Atomic relaxation was carried out until the Hellmann-Feynman forces were less than $0.03 \mathrm{eV} / \AA ̊$. . The ZnTeSe core (diameter $\mathrm{d}=36 \AA$ ) was generated by cutting out a zincblende (ZB) bulk structure, and cluster surfaces were passivated by hydrogen atoms with charge of 0.5 or 1.5 . The vacuum spacing between cluster surfaces was set as $10 \AA$, which was sufficient for minimising artificial interlayer interaction. The conduction band was up-shifted by $1.60 \mathrm{eV}$ to ensure that the calculated $\mathrm{ZnSe}$ bulk band gap $(1.12 \mathrm{eV})$ matched the experimental value $(2.72 \mathrm{eV})$. Even though the calculated band gap was considerably smaller than the experimental band gap, the localised Te states were accurately described by the density functional theory calculations. The Coulomb interaction energies between the electrons and holes of the ZnTeSe cores $(-0.140 \mathrm{eV}--0.139 \mathrm{eV}$ for $\mathrm{x}=0-0.07)$ were estimated using the effective mass approximation method.

Effective-mass approximation. The HOMO-LUMO energy gaps of the QDs were calculated using effective mass approximation. The electron effective masses of $\mathrm{ZnTe}, \mathrm{ZnSe}, \mathrm{ZnS}$ used in the calculation were $0.09,0.16$, and 0.39 ; the respective hole effective masses were $0.6,0.75$, and 1.76 ; the bulk energy band gaps were $2.26,2.72$, and $3.68 \mathrm{eV}$; and the relative permittivities were 10.3, 9.1, and 8.9, respectively. The overall effective mass and relative permittivity of the ZnTeSe cores was averaged by the mole fraction of the elemental components, and the bulk energy band gap was obtained from $E_{g}=2.72$ $1.837 x+1.450 x^{2}$. The valence band offset between the $\mathrm{ZnTeSe}$ cores with $3 \%, 5 \%$, and $7 \% \mathrm{Te}$ and $\mathrm{ZnSe}$ was calculated as $0.06 \mathrm{eV}, 0.10 \mathrm{eV}$, and $0.14 \mathrm{eV}$, respectively. An external electric field was applied by placing the QDs at the centre of a cylindrical vacuum space (diameter $=60 \mathrm{~nm}$, length $=100 \mathrm{~nm}$ ) in which both sides were composed of electrodes. The electric potential distribution in the QDs for various voltage differences between the electrodes was calculated by solving Laplace's equation using the finite element method implemented in the COMSOL Multiphysics software.

\section{Declarations}

Acknowledgments 
We thank S. Sul for assisting with spectroscopic measurements and Victor I. Klimov for helpful discussions.

\section{Author contributions}

E. J. directed the study and designed the experiments. E. J., T. K., and H. J. wrote the manuscript. S. C. performed the computational modelling. H. J. conducted the photo dynamic analysis. Y. W. prepared and characterised the QDs. J. L. and T. K. fabricated and characterised the devices.

\section{Competing interests statement}

The authors declare no competing interests.

\section{References}

1. Shirasaki, Y., Supran, G. J., Bawendi, M. G. \& Bulović, V. Emergence of colloidal quantum-dot lightemitting technologies. Nat. Photonics 7(1), 13-23 (2013). 10.1038/nphoton.2012.328

2. Yang, Z. et al. Recent advances in quantum dot-based light-emitting devices: Challenges and possible solutions. Mater. Today 24, 69-93 (2019). 10.1016/j.mattod.2018.09.002

3. Qi, H. et al. Research progress and challenges of blue light-emitting diodes based on II-VI semiconductor quantum dots. J. Mater. Chem. C 8(30), 10160-10173 (2020). 10.1039/D0TC02272A

4. Jia, H., Wang, F. \& Tan, Z. Material and device engineering for high-performance blue quantum dot light-emitting diodes. Nanoscale 12(25), 13186-13224 (2020). 10.1039/d0nr02074e, Pubmed:32614007

5. Chen, S. et al. On the degradation mechanisms of quantum-dot light-emitting diodes. Nat. Commun. 10(1), 765 (2019). 10.1038/s41467-019-08749-2, Pubmed:30770861

6. Tamang, S., Lincheneau, C., Hermans, Y., Jeong, S. \& Reiss, P. Chemistry of InP nanocrystal syntheses. Chem. Mater. 28(8), 2491-2506 (2016). 10.1021/acs.chemmater.5b05044

7. Kim, T. et al. Efficient and stable blue quantum dot light-emitting diode. Nature 586(7829), 385-389 (2020). 10.1038/s41586-020-2791-x, Pubmed:33057219

8. Gao, M. et al. Bulk-like ZnSe quantum dots enabling efficient ultranarrow blue light-emitting diodes. Nano Lett. 21(17), 7252-7260 (2021). 10.1021/acs.nanolett.1c02284, Pubmed:34428068

9. Dai, X. et al. Solution-processed, high-performance light-emitting diodes based on quantum dots. Nature 515(7525), 96-99 (2014). 10.1038/nature13829, Pubmed:25363773

10. Mashford, B. S. et al. High-efficiency quantum-dot light-emitting devices with enhanced charge injection. Nat. Photonics 7(5), 407-412 (2013). 10.1038/nphoton.2013.70

11. Bae, W. K. et al. Controlling the influence of Auger recombination on the performance of quantum-dot light-emitting diodes. Nat. Commun. 4, 2661 (2013). 10.1038/ncomms3661, Pubmed:24157692

12. Lim, J., Park, Y. S., Wu, K., Yun, H. J. \& Klimov, V. I. Droop-free colloidal quantum dot light-emitting diodes. Nano Lett. 18(10), 6645-6653 (2018). 10.1021/acs.nanolett.8b03457, Pubmed:30198267 
13. Lesnyak, V., Dubavik, A., Plotnikov, A., Gaponik, N. \& Eychmüller, A. One-step aqueous synthesis of blue-emitting glutathione-capped ZnSe 1-x Tex alloyed nanocrystals. Chem. Commun. (Camb) 46(6), 886-888 (2010). 10.1039/b919986a, Pubmed:20107639

14. Jang, E. P. et al. Synthesis of alloyed ZnSeTe quantum dots as bright, color-pure blue emitters. ACS Appl. Mater. Interfaces 11(49), 46062-46069 (2019). 10.1021/acsami.9b14763, Pubmed:31746194

15. Zhang, L., Lin, Z., Luo, J. W. \& Franceschetti, A. The birth of a Type-II nanostructure: Carrier localization and optical properties of isoelectronically doped CdSe:Te nanocrystals. ACS Nano 6(9), 8325-8334 (2012). 10.1021/nn303060r, Pubmed:22900638

16. Franzl, T. et al. CdSe:Te nanocrystals: Band-Edge versus Te-Related Emission. J. Phys. Chem. $C$ 111(7), 2974-2979 (2007). 10.1021/jp067166s

17. Kresse, G. \& Hafner, J., G. \& Hafner Ab initio molecular-dynamics simulation of the liquid-metalamorphous-semiconductor transition in germanium. Phys. Rev. B 49(20), 14251-14269 (1994). 10.1103/PhysRevB.49.14251

18. Norris, D. J. \& Bawendi, M. G. Measurement and assignment of the size-dependent optical spectrum in CdSe quantum dots. Phys. Rev. B Condens. Matter 53(24), 16338-16346 (1996). 10.1103/physrevb.53.16338, Pubmed:9983472

19. Lomascolo, M., Cretì, A., Leo, G., Vasanelli, L. \& Manna, L. Exciton relaxation processes in colloidal core/shell ZnSe/ZnS nanocrystals. Appl. Phys. Lett. 82(3), 418-420 (2003). 10.1063/1.1537050

20. Lad, A. D. \& Mahamuni, S. Effect of ZnS shell formation on the confined energy levels of ZnSe quantum dots. Phys. Rev. B 78(12) (2008). 10.1103/PhysRevB.78.125421, 125421

21. Deng, Y. et al. Deciphering exciton-generation processes in quantum-dot electroluminescence. Nat. Commun. 11(1), 2309 (2020). 10.1038/s41467-020-15944-z, Pubmed:32385262

22. Empedocles, S. A. \& Bawendi, M. G. Quantum-confined stark effect in single CdSe nanocrystallite quantum dots. Science 278(5346), 2114-2117 (1997). 10.1126/science.278.5346.2114, Pubmed:9405345

23. Shirasaki, Y., Supran, G. J., Tisdale, W. A. \& Bulović, V. Origin of efficiency roll-off in colloidal quantum-dot light-emitting diodes. Phys. Rev. Lett. 110(21), 217403 (2013). 10.1103/PhysRevLett.110.217403

24. Lee, T. et al. Bright and stable quantum dot light-emitting diodes. Adv. Mater., e2106276 (2021). 10.1002/adma.202106276, 34706113

25. Yu, P. et al. Absorption cross-section and related optical properties of colloidal InAs quantum dots. J. Phys. Chem. B 109(15), 7084-7087 (2005). 10.1021/jp046127i, Pubmed:16851806

26. Klimov, V. I. Optical nonlinearities and ultrafast carrier dynamics in semiconductor nanocrystals. J. Phys. Chem. B 104(26), 6112-6123 (2000). 10.1021/jp9944132

27. Avidan, A. \& Oron, D. Large blue shift of the Biexciton State in tellurium doped CdSe colloidal quantum dots. Nano Lett. 8(8), 2384-2387 (2008). 10.1021/nl801241m, Pubmed:18642954 


\section{Figure 1}

a
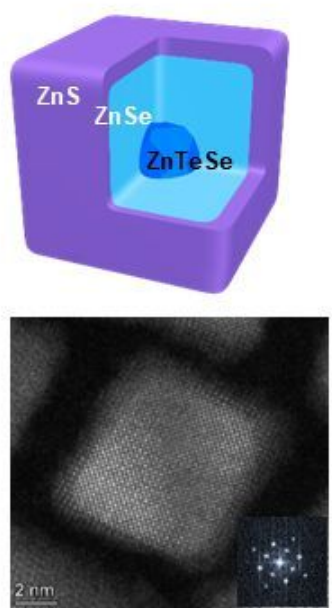

d

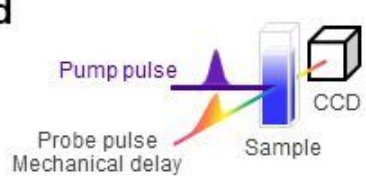

Mechanical dela

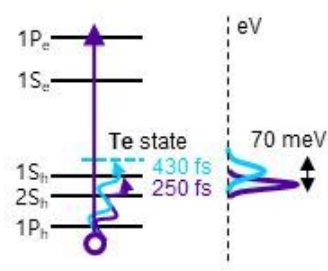

b

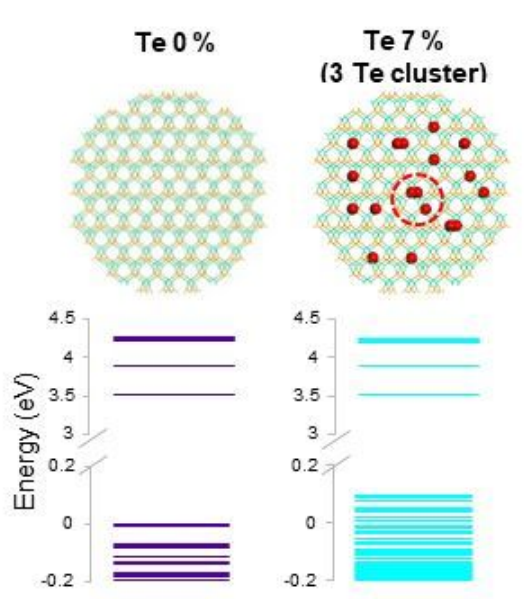

Te $7 \%$

(5 Te cluster)
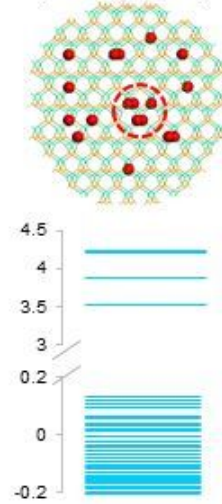

c

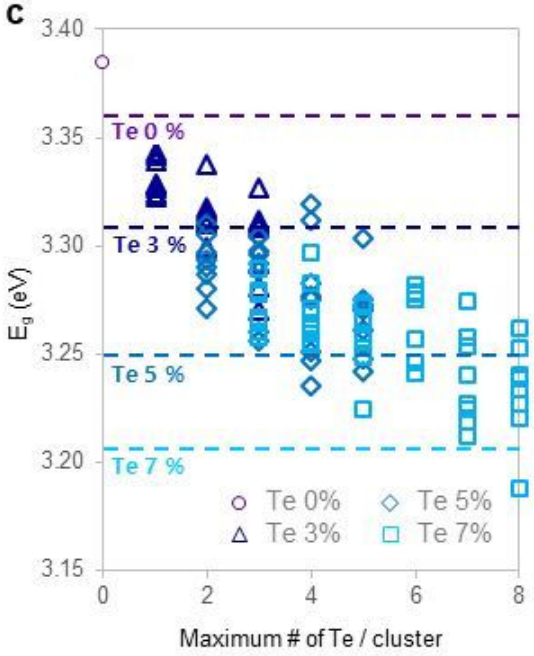

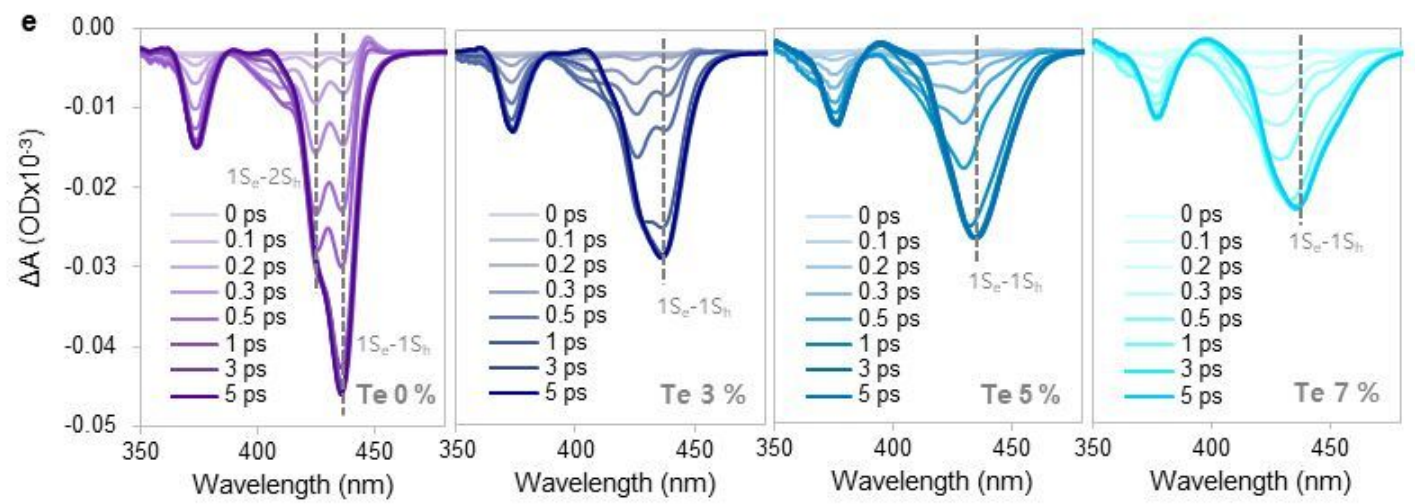

Figure 1

Electronic structure and absorption nature of ZnTeSe/ZnSe/ZnS QDs with a Te atomic cluster. (a)

Schematic of ZnTeSe/ZnSe/ZnS QD (top) and HR-TEM image (bottom) with a diffraction pattern in [110] direction. (b) Atomic structure models of the ZnTeSe cores used in the DFT calculation (top) and their calculated energy level diagrams (bottom). Two configurations of three atomic Te clusters and five atomic Te clusters in 7 \% Te model are denoted by red circles. (c) Experimental (dashed line) and calculated energy band gaps (symbol) for the ZnTeSe cores according to Te concentration and maximum number of Te atoms in each cluster. (d) Schematic of femtosecond TA measurement and the energy level diagram with the transitions for the QDs with $0 \%$ Te (250 fs) and $7 \%$ Te (430 fs). (e) Temporal evolutions of absorbance difference $(\Delta \mathrm{A})$ after photoexcitation at $400 \mathrm{~nm}$. 

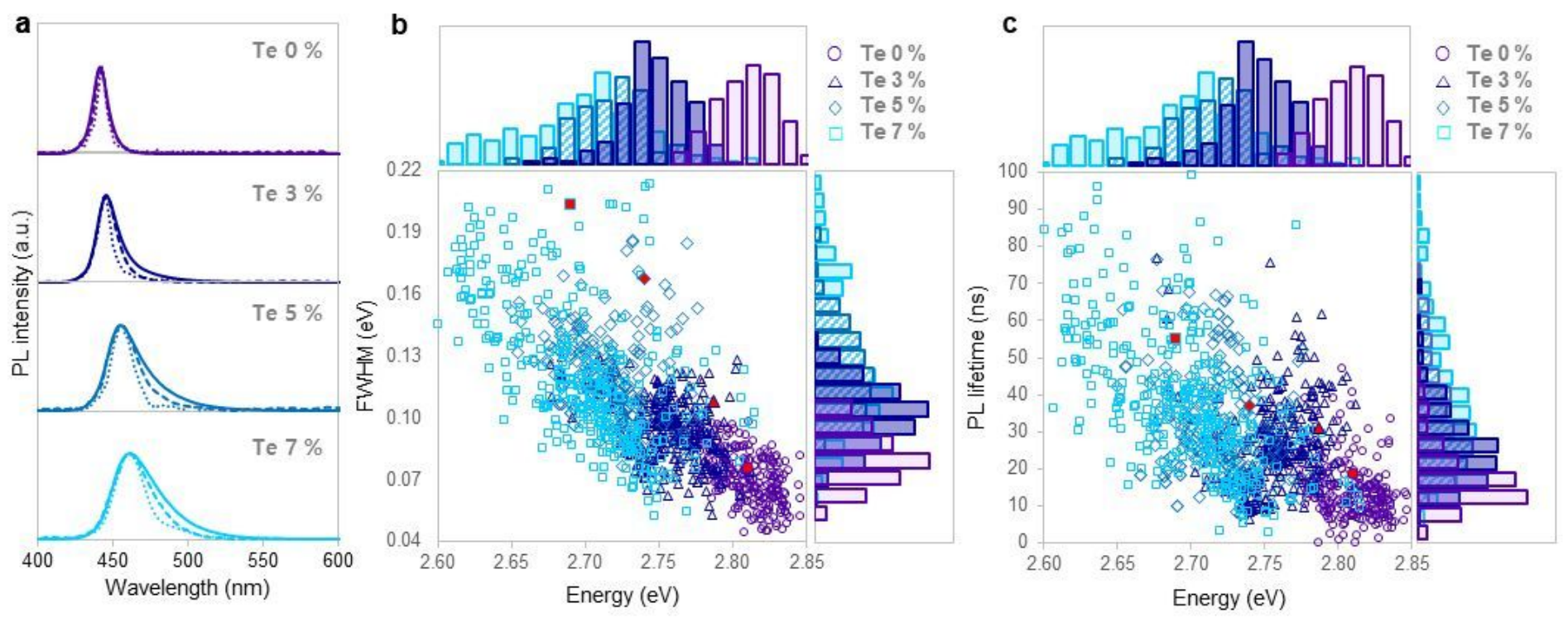

Figure 2

Single dot analysis. (a) Representative PL spectra of ensemble (solid), single dot (dashed), and single frame (1 s) (dotted) for ZnTeSe/ZnSe/ZnS QDs with 0 \%, 3 \%, 5 \%, and $7 \%$ Te. (b, c) Two-dimensional scatter plots of full width at half maximum (FWHM) and time-resolved PL decay lifetime of individual ZnTeSe/ZnSe/ZnS QDs as a function of emission peak energy. Red coloured solid symbols denote the ensemble data. Distributions for each value are presented as one-dimensional histograms over each axis. Statistical evaluations are summarised in Supplementary Table 2, and representative PL decay profiles of the single dots are presented in Supplementary Figure 7.
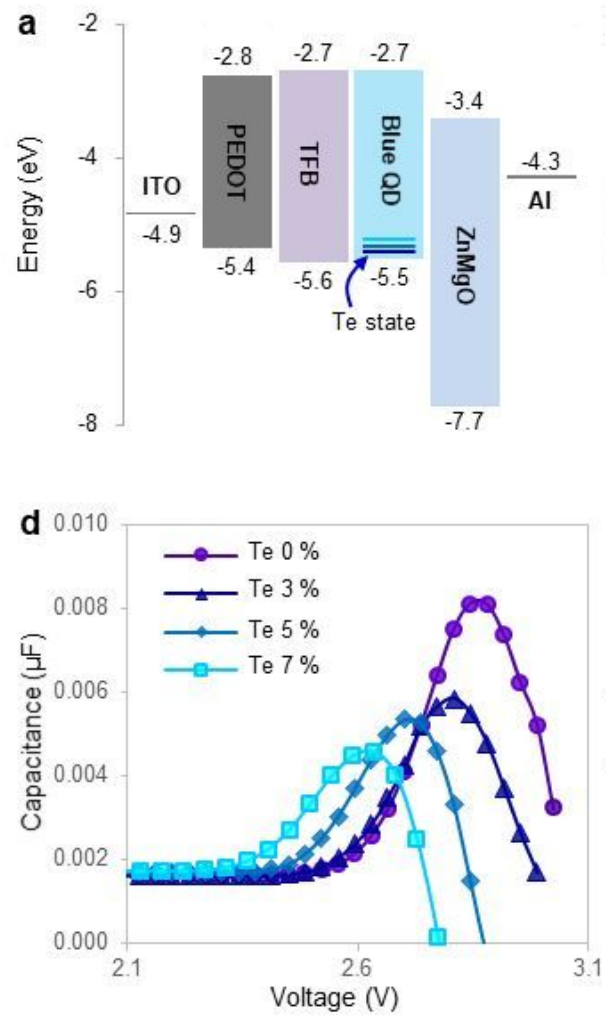
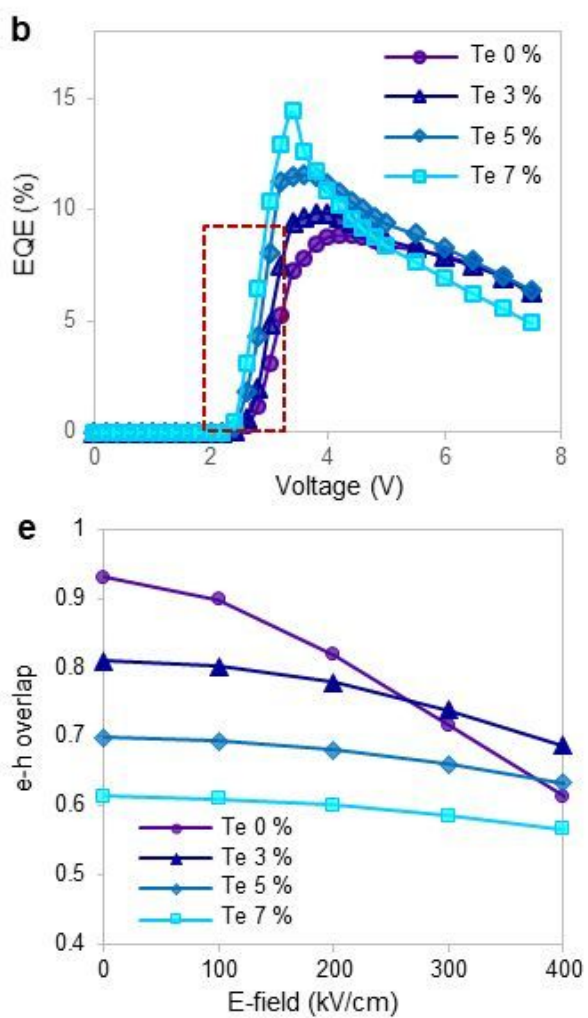
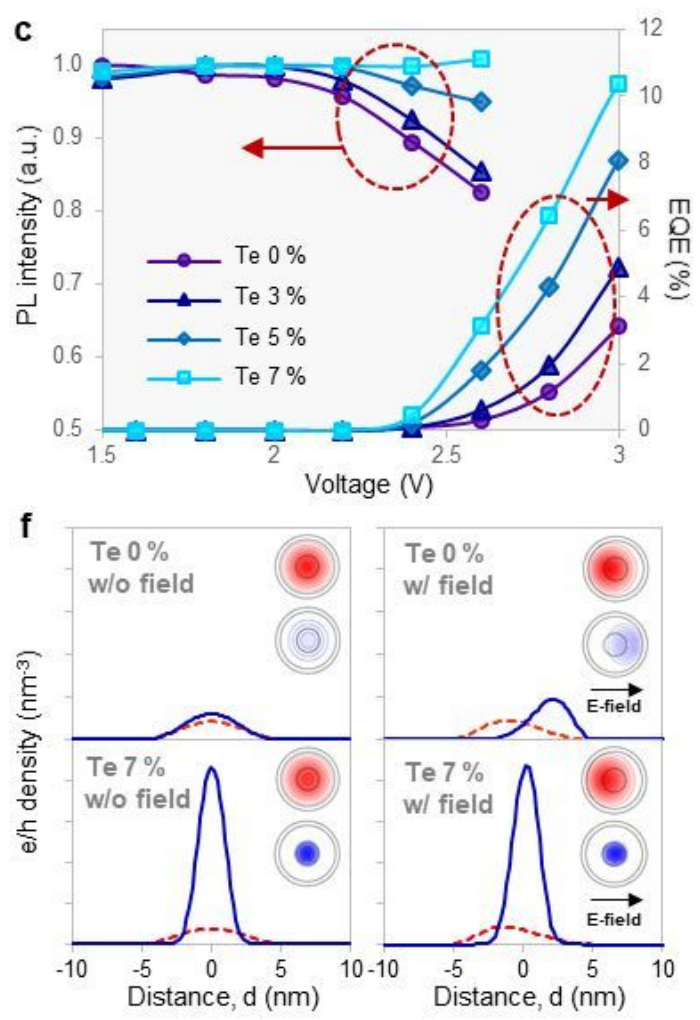


\section{Figure 3}

Characterisation of QD-LED devices. (a) Energy-band diagram of a device. (b) EQE-voltage profiles. (c) PL intensity and EQE of the devices over low voltages close to turn-on, which correspond to the red dashed box in Figure 3b. (d) Capacitance-voltage characteristics. (e) Calculated electron-hole overlap as a function of electric field. ( $f$ ) Distribution of electron (red dashed line) and hole (blue solid line) wave functions in the ZnTeSe/ZnSe/ZnS QDs with $0 \%$ and $7 \%$ Te with and without electric fields of 300 $\mathrm{kV} / \mathrm{cm}$. (Insets show e/h wave function contour plots) (ITO: indium tin oxide; PEDOT: poly(ethylenedioxythiophene):polystyrenesulfonate; TFB: poly(9,9-dioctyl-fluorene-co-N-(4-(3methylpropyl)) diphenylamine).

a

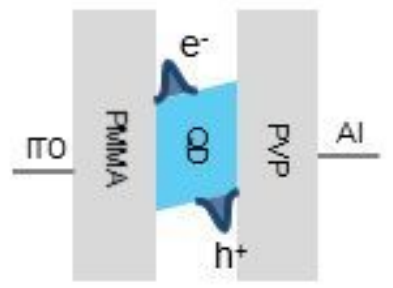

b

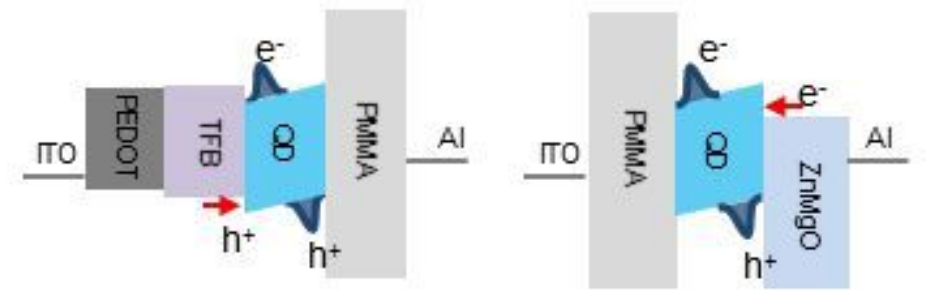

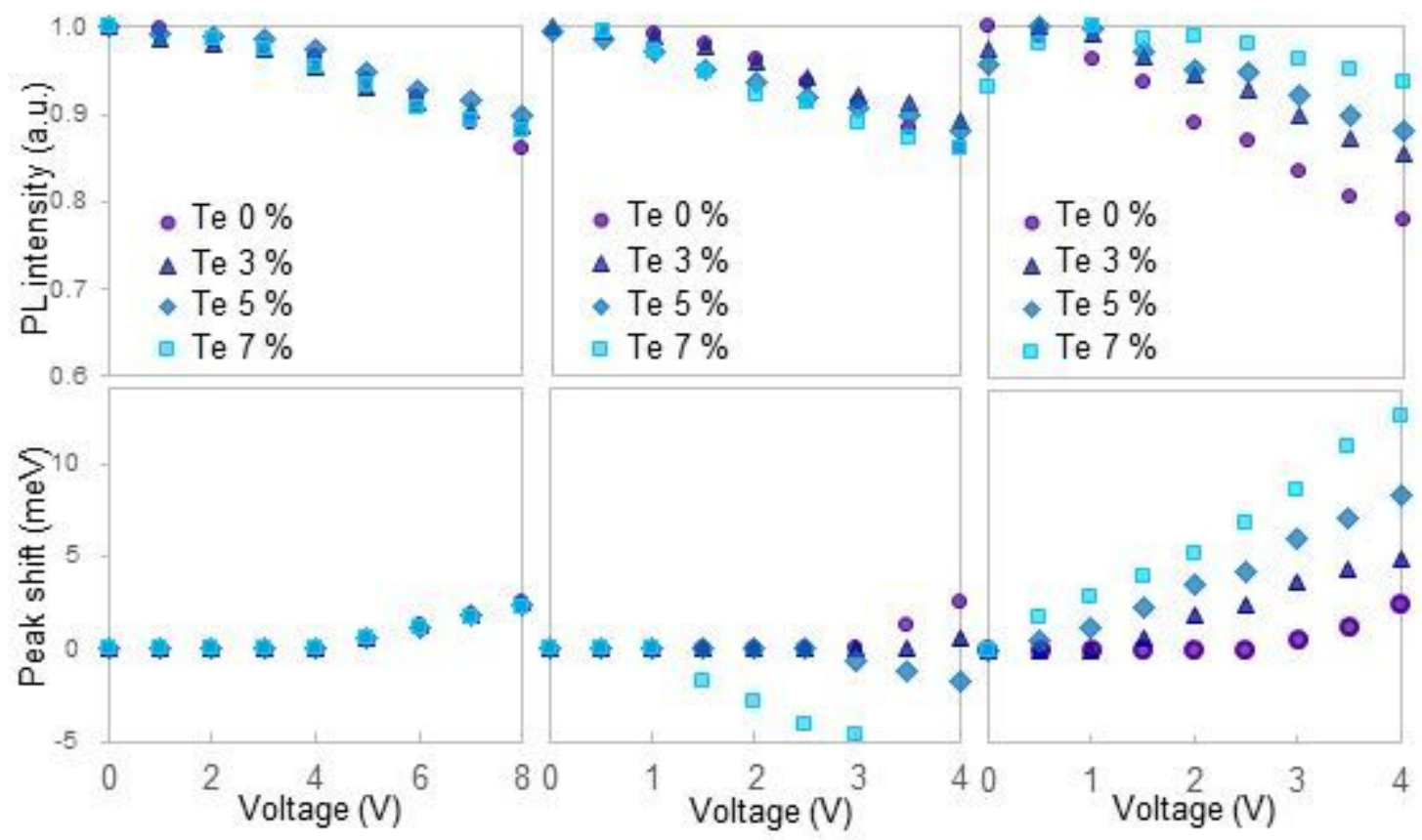

Figure 4

Characterisation of ZnTeSe/ZnSe/ZnS QD capacitor devices. Schematic of each structure (top), PL intensity according to voltage (middle), and PL peak shift according to voltage (bottom) for the device structures of (a) ITO/PMMA/QD/PVP/Al, (b) ITO/PEDOT/TFB/QD/PMMA/Al, and (c) ITO/PMMA/QD/ZnMgO/Al. Voltage-dependent PL spectra and numerical data are presented in Supplementary Figure 11 and Supplementary Table 3, respectively (ITO: indium tin oxide; PMMA: poly(methyl methacrylate); PVP: polyvinylpyrrolidone). 

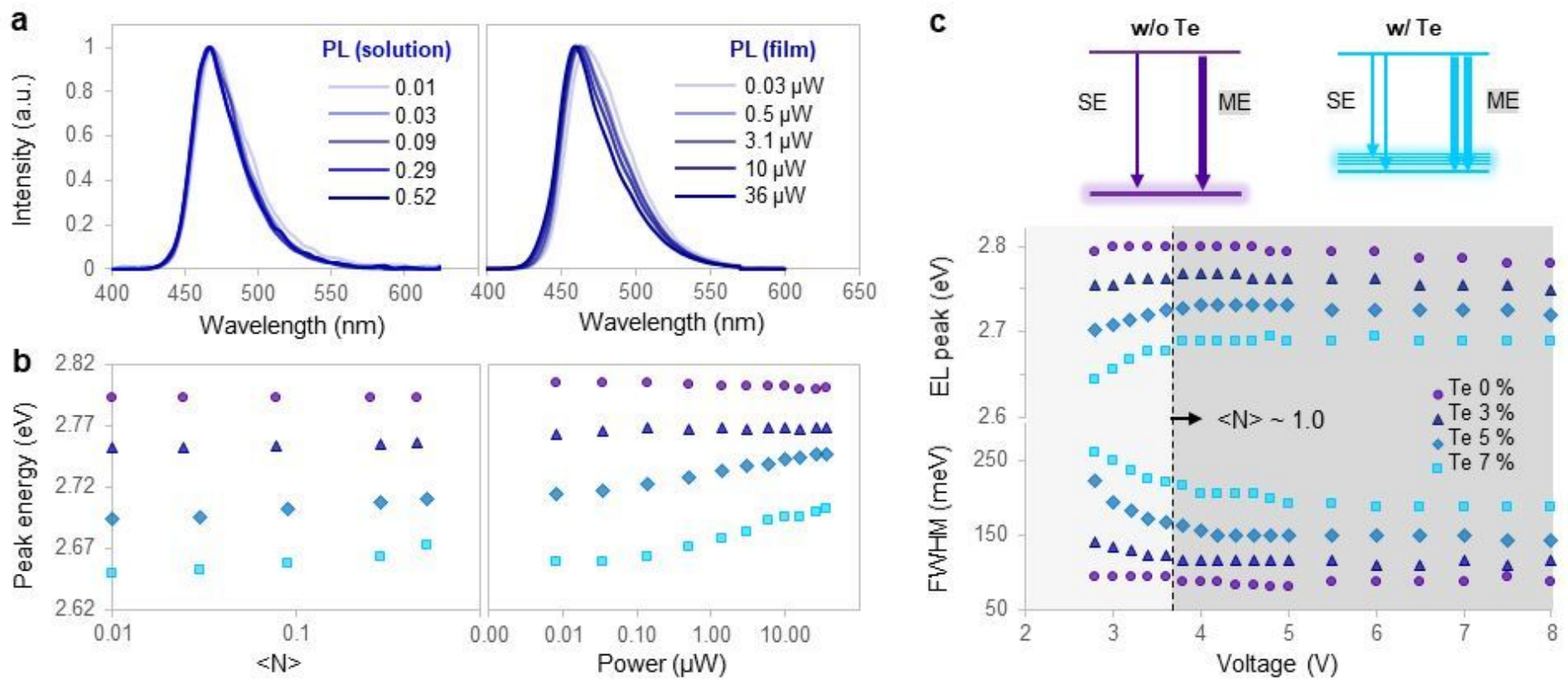

Figure 5

Effect of multi-excitons on the energy transition. (a) PL spectra of the solution as a function of excitation flux density (left) and the PL spectra of the film as a function of excitation laser power (right) for the ZnTeSe/ZnSe/ZnS QDs with $7 \%$ Te. (b) PL peak shifts of the solution depending on the excitation flux density (left) and the PL peak shifts of the film depending on the excitation laser power (right) for the ZnTeSe/ZnSe/ZnS QDs with $0 \%, 3 \%, 5 \%$, and $7 \%$ Te. (c) Energy transitions in single exciton and multiexciton regimes for the QDs without and with Te doping (top), and the changes in the EL peak and FWHM depending on the operation voltage of the QD-LEDs using ZnTeSe/ZnSe/ZnS QDs with $0 \%, 3 \%, 5 \%$, and $7 \%$ Te. The corresponding EL spectra are presented in Supplementary Figure 12 (SE: single exciton, ME: multi excitons).

\section{Supplementary Files}

This is a list of supplementary files associated with this preprint. Click to download.

- SupplementaryData1F.xlsx

- SupplementaryTedopedBlueQD2112171.docx 